\title{
Immunohistochemical-based molecular subtyping of colorectal carcinoma using maspin and markers of epithelial-mesenchymal transition
}

\author{
LAURA BANIAS $^{1}$, IOAN JUNG ${ }^{1}$, TIVADAR BARA ${ }^{2}$, ZSOLT FULOP $^{2}$, PATRICIA SIMU ${ }^{3}$, \\ IUNIUS SIMU $^{3}$, CATALIN SATALA ${ }^{1}$ and SIMONA GURZU ${ }^{1,4}$ \\ Departments of ${ }^{1}$ Pathology, ${ }^{2}$ Surgery and ${ }^{3}$ Radiology; ${ }^{4}$ Research Center (CCAMF)-Microscopy Laboratory, University of \\ Medicine, Pharmacy, Sciences and Technology, 'George Emil Palade', 540139 Targu-Mures, Romania
}

Received June 6, 2019; Accepted November 15, 2019

DOI: $10.3892 / 01.2019 .11228$

\begin{abstract}
The aim of the present study was to classify colorectal carcinoma (CRC) into molecular subtypes, based on immunohistochemical (IHC) assessments. A total of 112 CRC samples were molecularly classified based on the expression levels of epithelial-mesenchymal transition (EMT)-associated IHC markers. A total of three molecular subtypes were defined: Epithelial, membrane positivity for E-cadherin and $\beta$-catenin, negative for vimentin; mesenchymal, E-cadherin-negative, nuclear $\beta$-catenin- and vimentin-positive; and hybrid cases, epithelial tumor core and mesenchymal tumor buds. Most of the cases were diagnosed as moderately differentiated adenocarcinoma $(\mathrm{n}=89$; $79.46 \%)$. The majority of cases $(n=100 ; 89.28 \%)$ exhibited a mismatch repair proficient status (microsatellite stable CRCs). A predominance of epithelial-type $(n=51 ; 45.54 \%)$ and hybrid CRCs $(n=47 ; 41.96 \%)$ was observed, whereas a few cases $(n=14 ; 12.50 \%)$ were classified as mesenchymal-type CRCs. This molecular classification was associated with pathological stage $(\mathrm{P}<0.01)$, pT stage $(\mathrm{P}=0.04)$, pN stage $(\mathrm{P}<0.01)$, the grade of tumor budding $(\mathrm{P}=0.04)$, and maspin expression in both the tumor core $(\mathrm{P}=0.04)$ and the invasion front $(\mathrm{P}<0.01)$. The mesenchymal-type cases predominantly exhibited lymph node metastases, high-grade budding and a tendency towards maspin nuclear predominance. All epithelial-type cases with maspin-only expression $(n=18)$ were non-metastatic. Patients with CRC of the epithelial subtype and those with a lymph node ratio (LNR) $\leq 0.15$ presented the best overall survival,
\end{abstract}

Correspondence to: Professor Simona Gurzu, Department of Pathology, University of Medicine, Pharmacy, Sciences and Technology, 'George Emil Palade', 38 Ghe Marinescu Street, 540139 Targu-Mures, Romania

E-mail: simonagurzu@yahoo.com

Key words: colorectal cancer, molecular classification, immunohistochemistry, maspin, budding, epithelial-mesenchymal transition followed by those with hybrid and mesenchymal subtypes. Nuclear maspin positivity was more frequent in cases with a high-budding degree compared with those with a low-budding degree $(\mathrm{P}=0.03)$. The EMT-associated molecular classification of CRCs may be used to identify the most aggressive CRCs, which show a mesenchymal phenotype, high-budding degree, maspin nuclear positivity and lymph node metastases. The pN stage, LNR and budding degree of patients, which can be evaluated with maspin expression, remain the most important prognostic factors.

\section{Introduction}

Since the Tumor-Node-Metastasis classification, routinely used for the diagnosis of patients with colorectal carcinoma (CRC), is not adequate to plan appropriate targeted therapy, novel approaches need to be applied, based on the molecular profile of CRC cells (1). One proposal is the assessment of tumor budding as a result of active epithelial-mesenchymal transition (EMT), which is known to be a poor prognostic marker $(1,2)$. The current study presented a simple method of budding evaluation to support its introduction in conventional diagnosis.

The epithelial cells are marked by membrane adhesivity markers, such as E-cadherin and $\beta$-catenin, whereas the mesenchymal phenotype is indicated by the loss of membrane E-cadherin expression, the translocation of membrane-to-nuclear $\beta$-catenin positivity and the gain in positivity for mesenchymal markers, including Slug, Twist or vimentin (2-4).

Based on the EMT and molecular profile of CRC cells, several molecular classifications have been proposed for CRCs. In a consensus published in 2015 (1), four main consensus molecular subtypes (CMS) of CRC were identified: i) CMS1: Hypermutated cases with BRAF mutations, overexpression of proteins implicated in DNA mismatch repair and microsatellite instability, and tumors in which immune reaction is important; ii) CMS2: Epithelial subtype, WNT and MYC signaling pathways activation and chromosomally unstable; iii) CMS3: Epithelial subtype, with KRAS mutations and metabolic deregulation; and iv) 
CMS4: Mesenchymal subtype, with transforming growth factor $\beta$ activation stromal invasion, and development of new blood vessels. Combined features were suggested to be interpreted as transition phenotypes (1). This classification was demonstrated to have clinical impact, as mesenchymal subtype carcinomas exhibit a more unfavorable prognosis, a higher risk of systemic metastases and peritoneal carcinomatosis, and chemoresistance abilities (1-4).

In the present study, based on conventional histopathological assessment and immunohistochemical (IHC) staining, the term hybrid CRC was used for two of the aforementioned groups (epithelial and mesenchymal subtypes), as well as the transition phenotypes, which display both epithelial and mesenchymal features (1). To separate the three groups, the EMT-associated markers E-cadherin, $\beta$-catenin and vimentin were assessed in the tumor cells of tissues by IHC. Cases that displayed membrane E-cadherin and $\beta$-catenin expression and were vimentin-negative, in both the core and buds, were considered as the epithelial type, whereas mesenchymal CRCs were E-cadherin-negative, along with $\beta$-catenin membrane to nuclear translocation and vimentin positivity. Cases with transition phenotypes, which were mostly characterized by epithelial core and mesenchymal buds, were included in the group of mesenchymal CRCs.

As the EMT was suggested to be associated with the tumor-budding degree (5), this parameter is routinely evaluated in the daily diagnosis of CRC and considered as a negative prognostic marker, particularly for stage II cases $(6,7)$.

The originality of the present study consists of both the quantification of EMT in the tumor core vs. the invasion front (tumor buds) and the quantification method. Furthermore, a novel marker called maspin was used instead of the classic cytokeratin AE1/AE3 marker. Maspin was validated by the Department of Pathology, University of Medicine, Pharmacy, Sciences and Technology (Tirgu-Mures, Romania) (8-12), where this marker is used in the daily diagnosis of CRC.

Maspin is a serine protease, known to act as a tumor suppressor. However, the role of maspin is dependent on its subcellular localization (cytoplasm vs. nucleus) (8-12). Previous studies (8-12) have demonstrated that the cytoplasmic localization of maspin is an indicator of improved prognosis, whereas nuclear predominance may indicate a risk of local recurrence or lymph node metastases. The loss of maspin expression identified in some CRC specimen is an indicator of increased risk of distant metastases (10-12).

To the best of our knowledge, the association between maspin and EMT, or the possible prognostic value of its quantification in the tumor core vs. buds is unknown. The present study validated the method in daily clinical practice and used maspin as a marker for routine CRC diagnosis.

\section{Materials and methods}

Selection criteria. The Research Ethics Committee of the University of Medicine and Pharmacy of Targu-Mures, Romania approved the present observational retrospective study.

A total of 112 consecutive patients with CRC, who underwent surgical resection at the Emergency County
Hospital (Targu Mures, Romania), between January 2015 and December 2018, were included in the present study. Only those patients that survived for $\geq 4$ months following surgery were accounted. The database included a population of 73 males and 39 females (male:female ratio, 1.87:1) with a median age of $64.78 \pm 10.97$ years (range, $33-88$ years). None of the patients received chemo-radiotherapy prior to surgery. Consecutive cases with and without lymph node metastases (pN1/N2 and pN0, respectively) were included, whereas cases with computed tomography- or histology-proven systemic metastases (pM1) were excluded.

The follow-up of patients was performed between 4 and 39 months following surgery. The follow-up was done in person or over the phone, for patients who did not attend the periodic checks. Overall survival time (OS) was calculated based on the dates of surgery and mortality.

Routine histopathological assessment. Surgical specimens were histologically evaluated based on the eighth edition of the American Joint Committee on Cancer guidelines (13). In all cases, in addition to establishing the pTN stage and tumor grade, the lymph node ratio (LNR) and mismatch repair (MMR) status were determined in paraffin-embedded tissues. For study reliability, all IHC stains were analyzed by three pathologists independently.

Based on the number of metastatic lymph nodes, the cases were divided into three groups: i) pN0 (no metastases); ii) pN1 (metastases in one or two lymph nodes or the presence of tumor deposits); and iii) pN2 (more than two nodes with metastases). LNR was defined as the proportion of lymph nodes with metastases to the total number of lymph nodes. Cases were divided into two groups, using the LNR cutoff value of $\leq 0.15$ based on previously published data (14).

IHC reactions were performed from formalin-fixed paraffin-embedded tissues (fixed in $10 \%$ neutral buffered formalin), using the detection system EnVision ${ }^{\mathrm{TM}}$ FLEX (Dako; Agilent Technologies, Inc.). The histological sections, of 4-5 $\mu \mathrm{m}$ thickness, were deparaffinized and rehydrated, after which the activity of the endogenous peroxidase was blocked at room temperature by incubation with EnVision ${ }^{\mathrm{TM}}$ FLEX Peroxidase-Blocking reagent for $5 \mathrm{~min}$. Antigen retrieval consisted of incubating histological sections for 30-40 min at $100^{\circ} \mathrm{C}$ in citrate buffer $(0.01 \mathrm{M})$ with $\mathrm{pH} 6.0$ or high $\mathrm{pH}$ solution, depending on the antibody used (Table I). After incubation with the primary antibody (for $60 \mathrm{~min}$ at room temperature) and the secondary antibody (Dako EnVision ${ }^{\mathrm{TM}}$ FLEX/HRP detection reagent; $20 \mathrm{~min}$ at room temperature), the reactions were visualized by EnVision ${ }^{\mathrm{TM}}$ FLEX DAB+ Chromogen, followed by counterstaining with Mayer Hematoxylin for $1 \mathrm{~min}$ at room temperature. The stained slides were evaluated using a light microscope with magnifications ranging from $\mathrm{x} 10$ to $\mathrm{x} 40$. Clones and dilutions of the primary antibodies are included in Table I.

The MMR status was evaluated using four well-known IHC markers: mutL homolog 1 (MLH-1), mutS homolog 2 (MSH-2), PMS1 homolog 2, mismatch repair system component (PMS-2) and mutS homolog 6 (MSH-6) (Table I). The cases that showed nuclear positivity for all four markers were declared as having a microsatellite-stable status (MSS). If one of the markers was negative, the case was sent for molecular 
Table I. Antibodies used for immunohistochemical stains.

\begin{tabular}{llll}
\hline Antibody (company) & Clone (catalog nr/code) & Dilution & Antigen retrieval \\
\hline E-cadherin (Dako; Agilent Technologies, Inc.) & NCH-38 (M3612) & $1: 50$ & High retrieval solution (pH 10) \\
$\begin{array}{l}\text { 3-catenin (Dako Agilent Technologies, Inc.) } \\
\text { Vimentin (Dako; Agilent Technologies, Inc.) }\end{array}$ & V9-catenin-1 (IR702) & $1: 150$ & High retrieval solution (pH 10) \\
MLH1 (Leica Microsystems GmbH) & ES05 (MLH1-L-CE) & $1: 800$ & High retrieval solution (pH 10) \\
MSH2 (Leica Microsystems GmbH) & 25D12 (MSH2-CE) & $1: 50$ & High retrieval solution (pH 10) \\
PMS2 (Leica Microsystems GmbH) & M0R4G (PMS2-L-CE) & $1: 50$ & High retrieval solution (pH 10) \\
MSH6 (Leica Microsystems GmbH) & PU29 (MSH6-L-CE) & $1: 100$ & High retrieval solution (pH 10) \\
Maspin (Leica Microsystems GmbH) & EAW24 (MASPIN-CE) & $1: 50$ & Citrate (pH 6)
\end{tabular}

PCR examination of the microsatellite status, as previously reported (15).

Budding degree and maspin quantification. The quantification of tumor buds was based modifications on the criteria proposed by the International Tumor Budding Consensus Conference in $2016(6,7)$, as previously reported (8-12). 'Hotspot budding areas' were identified by light microscopy on histology slides stained with hematoxylin-eosin (HE) and Maspin at the invasion front, using a x10 objective lens. Subsequently, single tumor cells and clusters of no more than four tumor cells were counted with a $x 20$ objective lens $\left(0.785 \mathrm{~mm}^{2}\right.$ field area) and cases are classified as low- $(<5$ buds/hotspot) or high-grade budding ( $\geq 5$ buds/hotspot) $(6,7)$.

Maspin expression was evaluated in both the tumor core and the tumor buds. Maspin quantification was performed in 'hotspot budding areas', without taking into account the zones with inflammatory stroma or necrosis. Based on the dual cytoplasmic-nuclear expression of maspin in tumor cells and a cutoff value of $10 \%$ (Table II), cases were considered to be: negative (no stain); carcinomas with cytoplasm positivity (cytoplasmic positivity, without nuclear expression); or carcinomas with nuclear predominance, also known as carcinomas with dual positivity (nuclear + cytoplasmic) $(8,10,11)$.

EMT-based subtyping. As aforementioned, cases were classified into three subtypes of CRC, based on the expression levels of three EMT-associated IHC markers: E-cadherin, $\beta$-catenin and vimentin (Tables I and III). The three molecular CRC subtypes were defined as: Group A or epithelial (membrane E-cadherin/membrane $\beta$-catenin/negative vimentin, in the tumor core and buds); Group B or hybrid CRCs (epithelial immunoprofile in the tumor core and the mesenchymal profile of tumor buds); and Group $\mathrm{C}$ or mesenchymal (negative E-cadherin/nuclear $\beta$-catenin/vimentin-positive, in tumor core and buds)

Statistical analysis. Statistical analysis, both descriptive (indicating the median value and the standard deviation), as well as establishing correlations between the three molecular subtypes and the clinicopathological parameters, and between the grade of tumor budding and the IHC expression of Maspin, was performed using GraphPad Prism v7 software (GraphPad Software, Inc.). $\mathrm{P}<0.05$ was considered to indicate a statistically significant difference, calculated by the $\chi^{2}$ test. OS rate
Table II. Interpretation of maspin expression, according to its subcellular localization and percentage of positive cells.

Subcellular localization in tumor cells, \%

\begin{tabular}{lccl}
\hline Cytoplasm & Nuclei & Cell membrane & Interpretation \\
\hline$<10$ & $<10$ & No stain & Negative \\
$\geq 10$ & $<10$ & No stain & Cytoplasm \\
$\geq 10$ & $\geq 10$ & No stain & $\begin{array}{l}\text { Dual (nucleus + } \\
\text { cytoplasm) }\end{array}$ \\
\hline
\end{tabular}

was evaluated using Kaplan-Meier survival analysis and the log-rank (Mantel-Cox) test.

\section{Results}

Clinicopathological parameters. Most cases $(n=89)$ were diagnosed as moderately differentiated $(\mathrm{G} 2)$ adenocarcinoma, at pT3 or pT4 stages (96/112), without lymph node metastases $(\mathrm{n}=68)$, and exhibited a proficient MMR status - considered as MMS cases (100/112) (Table IV).

EMT-based subtyping and clinicopathological parameters. Based on the IHC expression of the EMT-associated markers, cases were classified into three molecular subtypes, presented in Fig. 1. More than one third of cases $(n=51)$ belonged to Group A (epithelial subtype) and a similar proportion $(n=47)$ to Group B (hybrid type). Fewer than $13 \%$ of cases $(n=14)$ were classified as Group C (mesenchymal type).

Molecular subtypes did not show any association with age, sex, tumor location, microscopic type or microsatellite status (Table IV). Although the statistical difference was not significant, due to a low number of cases, it is notable that all four G1 adenocarcinoma cases and all 12 low-grade microsatellite status (MSI-L) cases belonged to Group A.

A significant association was identified between the molecular groups and pathological stages $(\mathrm{P}<0.01)$, pT stage $(\mathrm{P}=0.04)$, $\mathrm{pN}$ stage $(\mathrm{P}<0.01), \mathrm{LNR}$ value $(\mathrm{P}<0.01)$ and the grade of tumor budding $(\mathrm{P}=0.04)$. Tumors with low- and high-budding grades are exemplified in Fig. 2. Only one non-metastatic case was classified in Group C; on the other hand, $>55 \%$ of cases with metastases in $>2$ lymph nodes or with an LNR value $>0.15$ 
Table III. Molecular classification of colorectal cancer specimens, according to subcellular localization and percentage of positive cells for epithelial-mesenchymal transition-associated markers.

\begin{tabular}{|c|c|c|c|c|c|c|c|}
\hline \multirow[b]{2}{*}{ Biomarker } & \multicolumn{3}{|c|}{ Tumor core $(\%)$} & \multicolumn{3}{|c|}{ Tumor buds (invasion front) (\%) } & \multirow[b]{2}{*}{ Interpretation } \\
\hline & Cytoplasm & Nuclei & Cell membrane & Cytoplasm & Nuclei & Cell membrane & \\
\hline E-cadherin & No stain & No stain & $\geq 10$ & No stain & No stain & $\geq 10$ & Group A or epithelial-type \\
\hline$\beta$-catenin & No stain & No stain & $\geq 10$ & No stain & No stain & $\geq 0$ & carcinoma \\
\hline Vimentin & No stain & No stain & No stain & No stain & No stain & No stain & \\
\hline E-cadherin & No stain & No stain & $\geq 10$ & No stain & No stain & $<10$ & Group B or hybrid-type \\
\hline$\beta$-catenin & No stain & No stain & $\geq 10$ & No stain & $\geq 10$ & $<10$ & carcinoma \\
\hline Vimentin & No stain & No stain & No stain & $\geq 10$ & No stain & No stain & \\
\hline E-cadherin & No stain & No stain & $<10$ & No stain & No stain & $<10$ & Group C or mesenchymal- \\
\hline$\beta$-catenin & No stain & $\geq 10$ & $<10$ & No stain & $\geq 10$ & $<10$ & type carcinoma \\
\hline Vimentin & $\geq 10$ & No stain & No stain & $\geq 10$ & No stain & No stain & \\
\hline
\end{tabular}

were also classified in Group C (Table IV). Cases from Groups A and $B$ exhibited a similar distribution of buds, whereas the highest proportion of high-grade budding cases were included in Group C (Table IV).

EMT-based subtyping and maspin expression. Maspin expression was considered to be negative, cytoplasmic or presenting dual expression (Fig. 3). An association between the molecular subgroups and maspin expression was observed in the tumor core $(\mathrm{P}=0.04)$, and more significantly $(\mathrm{P}<0.01)$ in the tumor buds (Table IV). In the tumor core, Group A predominantly exhibited cytoplasm-only expression, which was also found in the buds, in 18 of the 33 cases; in the other 15 cases, loss of maspin $(n=2)$ or mixed expression $(n=13)$ was seen in the tumor buds cells (Table IV). All of the 18 cases with cytoplasm-only positivity in both the core and buds were low-grade budding pN0 cases. In Group B, cytoplasm-only expression in the core was mainly transcripted in nuclear co-localization (dual expression) in the buds. In Group C, only four of the 14 cases exhibited cytoplasm-only expression in the core; however, purecytoplasmic positivity was not seen in the buds. Most of the cases in Group C showed dualmaspin subcellular expression (cytoplasm + nuclei; Table IV).

Dualmaspin positivity was significantly more frequent in the buds of the high-budding tumors compared with those in low budding tumors $(\mathrm{P}=0.03)$. In the budding areas, a significant cytoplasm (tumor core) to nucleus (invasion front) translocation was observed in both the low-budding $(\mathrm{P}<0.01)$ and high-budding cases $(\mathrm{P}<0.01$; Table $\mathrm{V})$.

The association of clinicopathological parameters and maspin immunostaining with the three molecular subtypes demonstrated that cases from Group $\mathrm{C}$ showed a high-budding degree and a more significant cytoplasm-to-nucleus translocation of maspin in tumor buds (Table IV).

If cytoplasmic-only maspin expression was an indicator of non-metastatic cases from Group A, maspin negativity did not prove to be an indicator of a certain molecular subgroup.

EMT-based subtyping and OS rate. Patients included in Group A presented the best OS rate, followed by those from
Groups B and C (Fig. 4); however, due to the low number of cases, this association was not statistically significant.

As previously demonstrated (14), patients with LNR $\leq 0.15$ had an improved OS, compared with those having an LNR $>0.15$ ( $\mathrm{P}=0.04$; Fig. 4). Except for the LNR value $(\mathrm{P}=0.04$; Fig. 4) and $\mathrm{pN}$ stage ( $\mathrm{P}=0.04$; Fig. 5), which proved in the present study to be indicators of OS, none of the examined clinicopathological parameters were independent prognostic factors.

Although an independent evaluation of maspin, E-cadherin and $\beta$-catenin was performed separately in the core and at the front, none of the IHC markers were identified as independent prognostic factors.

\section{Discussion}

Similar to the present study, a previous study was also mainly based on the microsatellite status and the EMT immunophenotype (1). However, several markers have been put forward for use. In some studies, the epithelial phenotype was identified using cytokeratin, caudal type homeobox 2, mucin-2 and trefoil factor 3, whereas, for confirmation of a mesenchymal subtype, FERM domain-containing protein 6 , cystic fibrosis transmembrane conductance regulator, zinc finger E-box-binding homeobox 1 or 5-hydroxytryptamine receptor 2B staining were evaluated, the latter subtype exhibiting worse prognosis $(1,16,17)$.

In the present study, IHC was used to evaluate the microsatellite status and to classify the cases as epithelial or mesenchymal by interpreting the expression of E-cadherin, $\beta$-catenin and vimentin. Specific antibodies were used for the identification of a transition subtype CRC, referred to as the hybrid type, which exhibited an epithelial core and mesenchymal buds. Based on the OS analysis and the association with lymph node metastasis, the mesenchymal subtype was identified to have the worst prognosis among the three categories. The significance of the association may be demonstrated in larger cohorts.

According to previous studies $(14,18,19)$ LNR and pN stage proved to be independent prognostic indicators of OS for metastatic and non-metastatic CRCs, using the cutoff value of 
Table IV. Clinicopathological features of colorectal carcinoma cases, according to their tumor molecular subtype (multiple associations).

\begin{tabular}{|c|c|c|c|c|c|}
\hline \multirow[b]{2}{*}{ Parameters } & \multirow[b]{2}{*}{$\begin{array}{l}\text { Total number } \\
\text { of cases } \\
(\mathrm{n}=112), \mathrm{n}(\%)\end{array}$} & \multicolumn{3}{|c|}{ Molecular subtypes } & \multirow[b]{2}{*}{ P-value } \\
\hline & & $\begin{array}{c}\text { Group A, } \\
\text { epithelial } \\
(\mathrm{n}=51), \mathrm{n}(\%)\end{array}$ & $\begin{array}{c}\text { Group B, } \\
\text { hybrid } \\
(\mathrm{n}=47), \mathrm{n}(\%)\end{array}$ & $\begin{array}{c}\text { Group C, } \\
\text { mesenchymal } \\
(\mathrm{n}=14), \mathrm{n}(\%)\end{array}$ & \\
\hline Age, years & & & & & 0.37 \\
\hline$<64$ & $45(40.2)$ & $20(39.2)$ & $17(36.2)$ & $8(57.1)$ & \\
\hline$\geq 64$ & $67(59.8)$ & $31(60.8)$ & $30(63.8)$ & $6(42.9)$ & \\
\hline Sex & & & & & 0.51 \\
\hline Female & $39(34.8)$ & $18(35.3)$ & $18(38.3)$ & $3(21.4)$ & \\
\hline Male & $73(65.2)$ & $33(64.7)$ & $29(61.7)$ & $11(78.6)$ & \\
\hline Tumor location & & & & & 0.64 (proximal + \\
\hline Proximal colon & $40(35.7)$ & $21(41.2)$ & $15(31.9)$ & $4(28.6)$ & distal colon \\
\hline Distal colon & $39(34.8)$ & $18(35.3)$ & $15(31.9)$ & $6(42.8)$ & vs. rectum) \\
\hline Upper rectum & $33(29.5)$ & $12(23.5)$ & $17(36.2)$ & $4(28.6)$ & \\
\hline Microscopic aspect & & & & & $0.39(\mathrm{G} 1+\mathrm{G} 2 \mathrm{vs}$ \\
\hline G1 & $4(3.6)$ & $4(7.8)$ & $0(0.0)$ & $0(0.0)$ & mucinous) \\
\hline G2 & $89(79.5)$ & $39(76.5)$ & $40(85.1)$ & $10(71.4)$ & \\
\hline G3 & $8(7.1)$ & $5(9.8)$ & $2(4.3)$ & $1(7.1)$ & \\
\hline Mucinous adenocarcinoma & $11(9.8)$ & $3(5.9)$ & $5(10.6)$ & $3(21.5)$ & \\
\hline Microsatellite status & & & & & 0.37 (MSS vs. \\
\hline MSS & $100(89.3)$ & $46(90.2)$ & $43(91.5)$ & $11(78.6)$ & MSI-L) \\
\hline MSI-L & $12(10.7)$ & $5(9.8)$ & $4(8.5)$ & $3(21.4)$ & \\
\hline pT stage & & & & & $0.04(\mathrm{pT} 1+2$ vs. \\
\hline 1 & $5(4.5)$ & $3(5.9)$ & $2(4.3)$ & $0(0.0)$ & pT3 + 4) \\
\hline 2 & $11(9.8)$ & $6(11.8)$ & $5(10.6)$ & $0(0.0)$ & \\
\hline 3 & $75(66.9)$ & $35(68.6)$ & $32(68.1)$ & $8(57.1)$ & \\
\hline 4 & $21(18.8)$ & $7(13.7)$ & $8(17.0)$ & $6(42.9)$ & \\
\hline pN stage & & & & & $<0.01$ (pN0 vs. \\
\hline 0 & $68(60.7)$ & $38(74.5)$ & $29(61.7)$ & $1(7.1)$ & $\mathrm{pN} 1+\mathrm{pN} 2)$ \\
\hline 1 & $28(25.0)$ & $10(19.6)$ & $13(27.7)$ & $5(35.7)$ & \\
\hline 2 & $16(14.3)$ & $3(5.9)$ & $5(10.6)$ & $8(57.2)$ & \\
\hline LNR & & & & & $<0.01(\leq 0.15$ \\
\hline$\leq 0.15$ & $85(75.9)$ & $46(90.2)$ & $38(80.9)$ & $1(7.1)$ & vs. $>0.15)$ \\
\hline$>0.15$ & $27(24.1)$ & $5(9.8)$ & $9(19.1)$ & $13(92.9)$ & \\
\hline Pathological stages & & & & & $<0.01$ (stage I + \\
\hline $\mathrm{I}$ & $8(7.1)$ & $5(9.8)$ & $3(6.4)$ & $0(0.0)$ & II vs. III) \\
\hline II & $60(53.6)$ & $33(64.7)$ & $26(55.3)$ & $1(7.1)$ & \\
\hline III & $44(39.3)$ & $13(25.5)$ & $18(38.3)$ & $13(92.9)$ & \\
\hline Grade of tumor budding & & & & & 0.04 (low vs. \\
\hline Low & $59(52.7)$ & $30(58.8)$ & $25(53.2)$ & $4(28.6)$ & high grade) \\
\hline High & $53(47.3)$ & $21(41.2)$ & $22(46.8)$ & $10(71.4)$ & \\
\hline Maspin expression-tumor core & & & & & 0.04 (negative vs. \\
\hline Negative & $22(19.6)$ & $9(17.6)$ & $9(19.1)$ & $4(28.6)$ & cytoplasm vs. dual \\
\hline Cytoplasm & $59(52.7)$ & $33(64.8)$ & $22(46.8)$ & $4(28.6)$ & expression) \\
\hline Nucleus + cytoplasm & $31(27.7)$ & $9(17.6)$ & $16(34.1)$ & $6(42.8)$ & \\
\hline Maspin expression-tumor buds & & & & & $<0.01$ (negative vs \\
\hline Negative & $22(19.6)$ & $11(21.6)$ & $7(14.9)$ & $4(28.6)$ & cytoplasm vs. dual \\
\hline Cytoplasm & $22(19.6)$ & $18(35.3)$ & $4(8.5)$ & $0(0.0)$ & expression) \\
\hline Nucleus + cytoplasm & $68(60.8)$ & $22(43.1)$ & 36 (76.6) & $10(71.4)$ & \\
\hline
\end{tabular}

pT stage, pathological T stage; pN stage, pathological N stage. 
Table V. Subcellular localization of maspin in the tumor core vs. the invasion front, associated with the tumor budding grade.

Grade of tumor budding

\begin{tabular}{|c|c|c|c|c|c|}
\hline \multirow{3}{*}{$\begin{array}{l}\text { Maspin } \\
\text { subcellular } \\
\text { localization }\end{array}$} & \multirow{2}{*}{\multicolumn{2}{|c|}{ Low budding $(n=59)$}} & \multirow{2}{*}{\multicolumn{2}{|c|}{ High budding $(n=53)$}} & \multirow[b]{3}{*}{ P-value } \\
\hline & & & & & \\
\hline & $\begin{array}{c}\text { 1. Tumor core, } \\
\text { n }(\%)\end{array}$ & $\begin{array}{c}\text { 2. Invasion front, } \\
\mathrm{n}(\%)\end{array}$ & $\begin{array}{c}\text { 3. Tumor core, } \\
\text { n }(\%)\end{array}$ & $\begin{array}{c}\text { 4. Invasion front, } \\
\mathrm{n}(\%)\end{array}$ & \\
\hline Negative & $13(22.1)$ & $14(23.7)$ & $9(17.0)$ & $8(15.1)$ & 1 vs. $2:<0.01 ; 1$ vs. $3: 0.19$ \\
\hline Cytoplasm & $34(57.6)$ & $16(27.1)$ & $25(47.2)$ & $6(11.3)$ & 2 vs. $4: 0.03 ; 3$ vs. $4:<0.01$ \\
\hline Nucleus + cytoplasm & $12(20.3)$ & $29(49.2)$ & $19(35.8)$ & $39(73.6)$ & \\
\hline
\end{tabular}

A

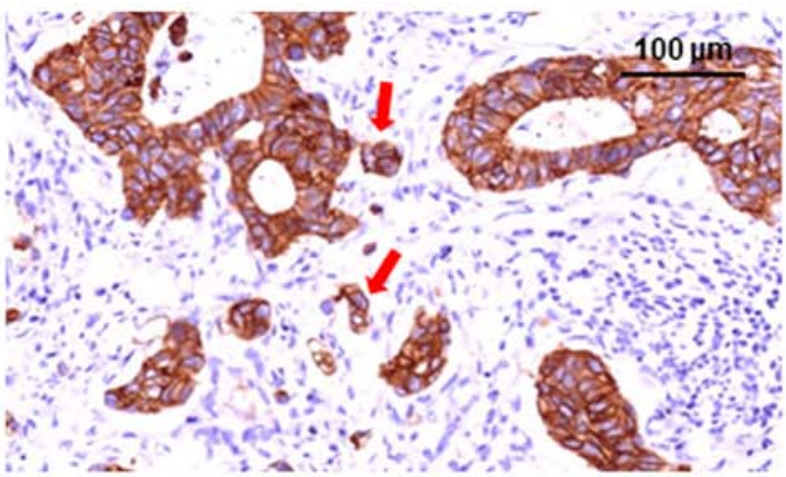

C

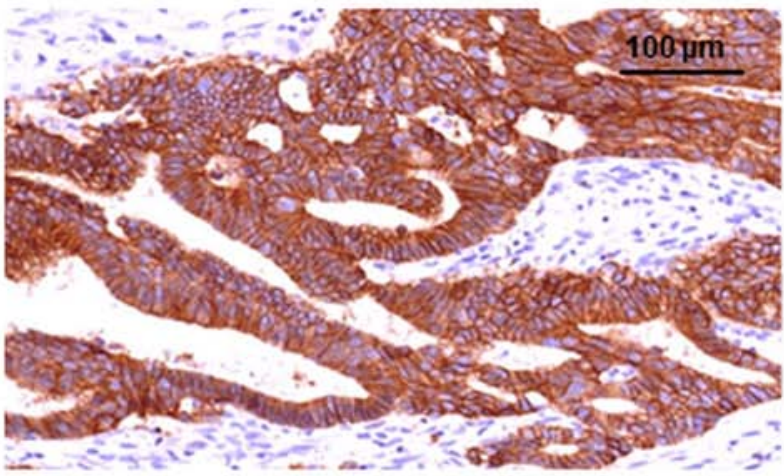

E

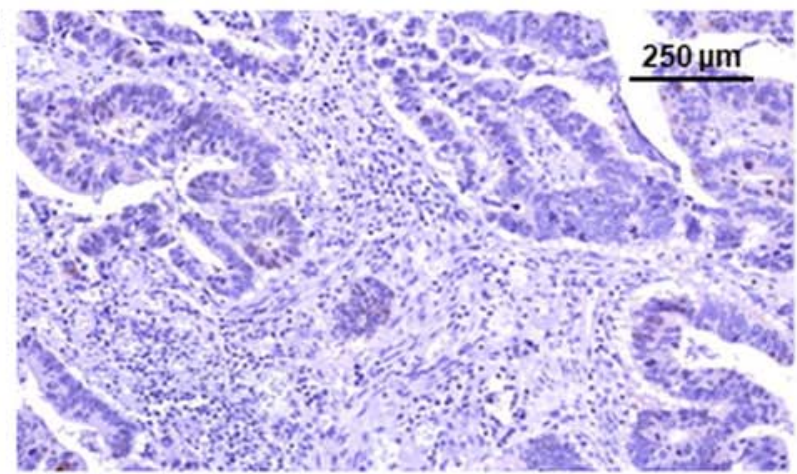

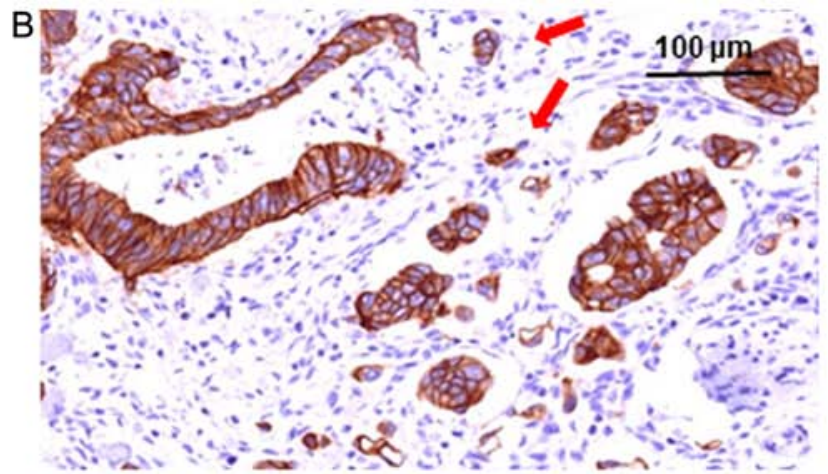
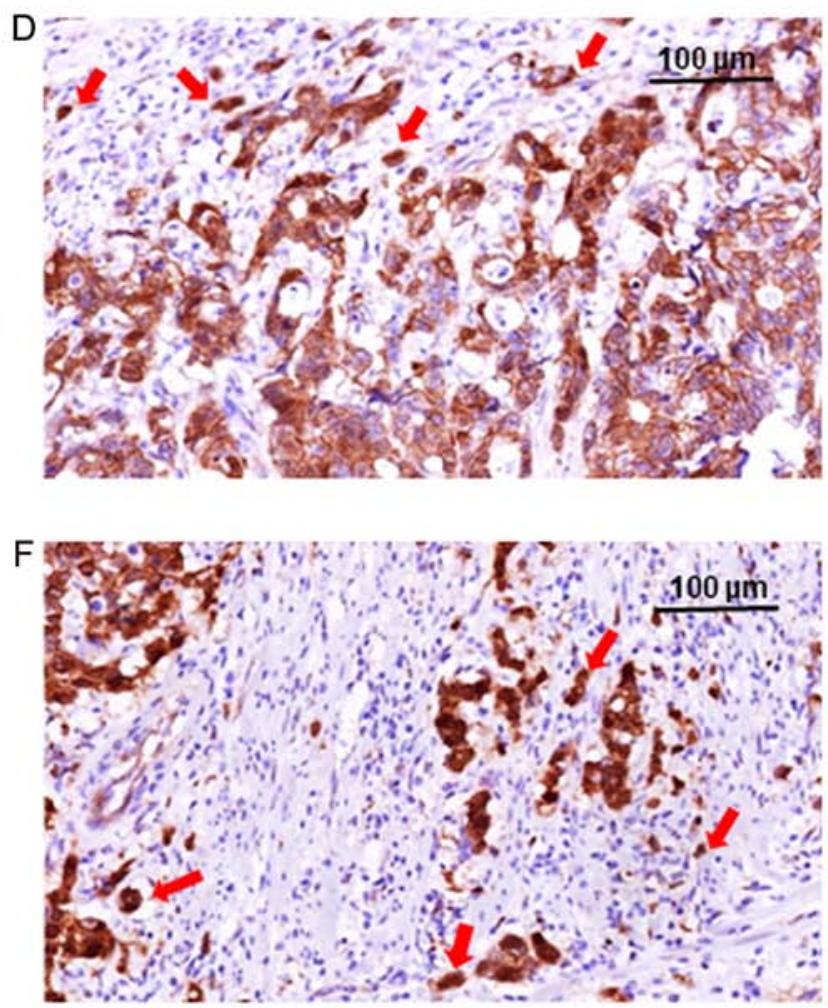

Figure 1. Molecular classification of colorectal cancer based on the immunohistochemical profile of E-cadherin and $\beta$-catenin. The epithelial subtype is characterized by diffuse membrane staining for both (A) E-cadherin and (B) $\beta$-catenin in core and buds, which are indicated by arrows. Hybrid subtype shows epithelial tumor center, with (C) E-cadherin and (D) $\beta$-catenin membrane expression, and mesenchymal buds, defined by nuclear $\beta$-catenin and marked by arrows. The mesenchymal subtype does not express (E) E-cadherin, and (F) $\beta$-catenin is predominantly nuclear, in both core and buds; the $\beta$-catenin nuclear positivity is indicated by arrows $(\mathrm{F})$

$\leq 0.15$. As the mesenchymal-type CRCs show a higher risk for lymph node metastases, independently of the pT stage, such cases should benefit from chemotherapy, even in the absence of lymph node metastases. 

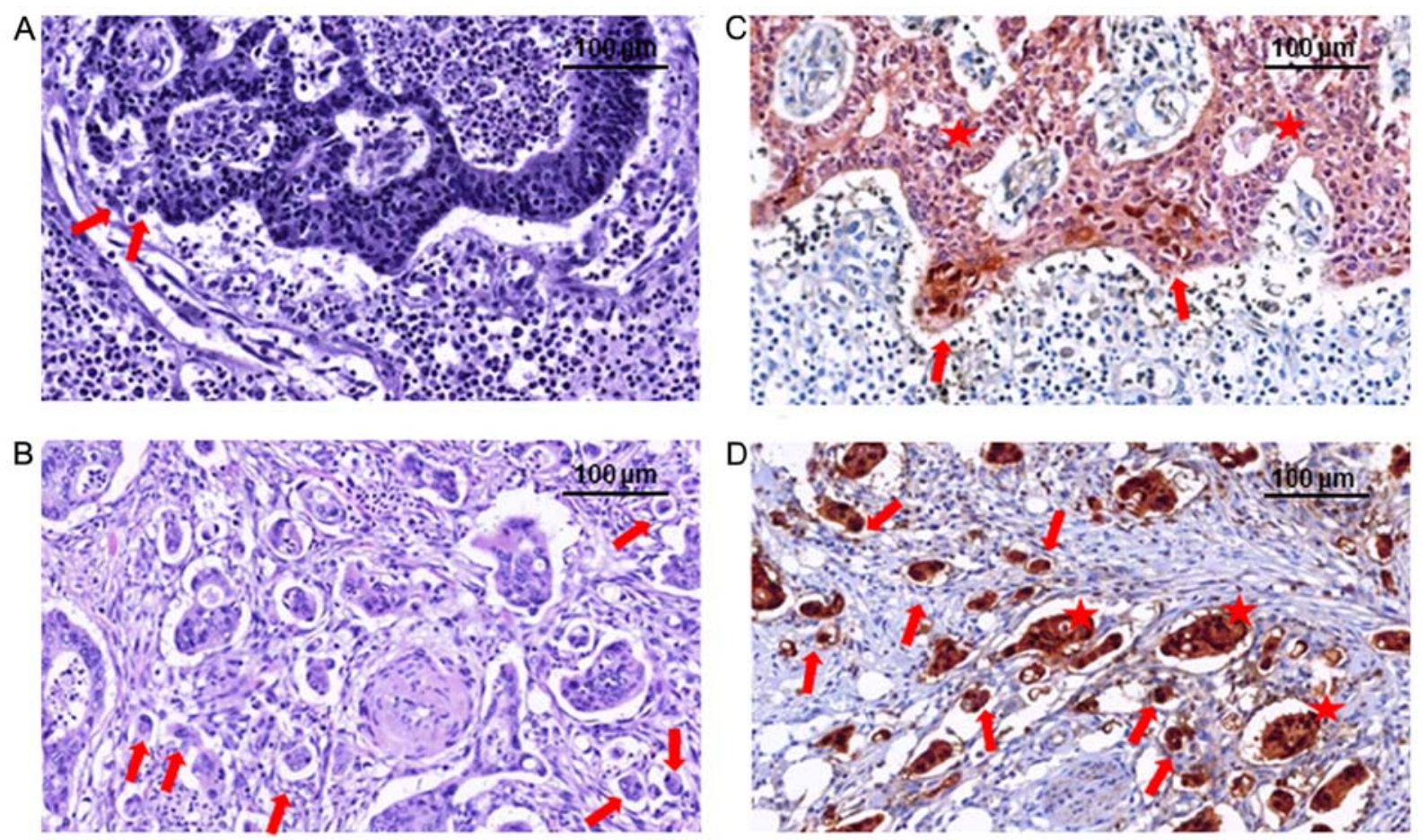

Figure 2. Classification of budding degree of colorectal carcinoma. (A) Low- and (B) high- budding cases can be seen with hematoxylin-eosin staining. (C) In low-budding cases, in the tumor core, maspin can be observed in the cytoplasm, with nuclear translocation in the invasion front/buds. (D) In high-budding cases, dual expression of maspin (nucleus + cytoplasm) is present in both the tumor core and buds. Tumor center/core is highlighted with stars and the buds are indicated with arrows. Scale bar, $100 \mu \mathrm{m}$.
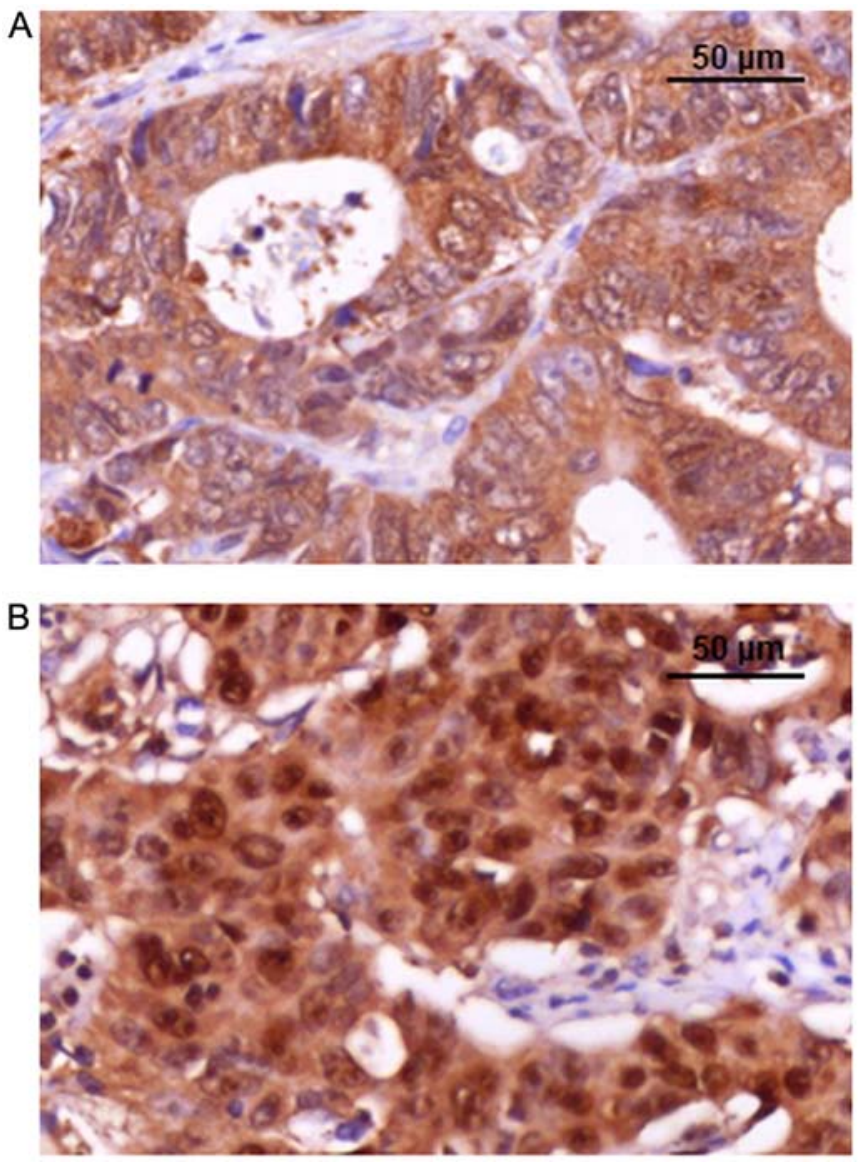

Figure 3. Maspin subcellular localization. (A) Cytoplasm positivity, without nuclear expression. (B) Dual positivity (nucleus + cytoplasm). Scale bar, $50 \mu \mathrm{m}$.
The present study proposed a simple method of budding evaluation, to support its introduction in conventional diagnosis, as a valuable prognostic parameter. This method was based on the quantification of maspin and can be easily used in routine practice.

The present study demonstrated the potential prognostic value of tumor buds, high-grade budding indicating lower OS, decreased disease-free and relapse-free survival, and association with advanced tumor stages and the nuclear localization of maspin $(6,8,10,20,21)$.

For patients with stage II and III CRC, the nuclear expression of maspin has been demonstrated to predict sensitivity to 5 -fluorouracil and levamisole $(9,12,22)$. In addition to the findings of the present study, it can be postulated that patients with MSS-CRCs diagnosed with stage II or III, with high-grade budding and nuclear maspin predominance, involving mainly mesenchymal-type CRCs, may be responders in the case of adjuvant chemotherapy (21). On the other hand, patients with mesenchymal-type CRCs may benefit from anti-EMT-associated agents, which are currently being tested in clinical trials $(4,23)$.

The present study underlined the necessity for tumor budding assessment in CRC specimens. The identification of cases with dual maspin expression (cytoplasm + nuclei) and high-grade budding, along with those, which show a mesenchymal phenotype, may have a prognostic and predictive impact for the implementation of anti-EMT-based clinical trials.

Although based on a small number of cases, the previous experience of the authors' team in the field of EMT and maspin guarantees credibility $(2,5,8-12)$. For study reliability, classic slides were used, not tissue microarrays. 
A

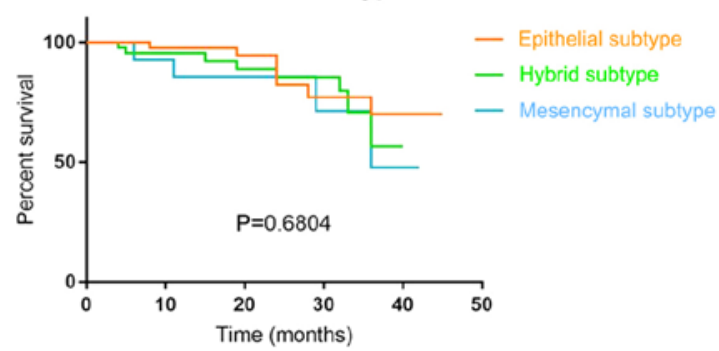

C Maspin subcellular localization - invasion front

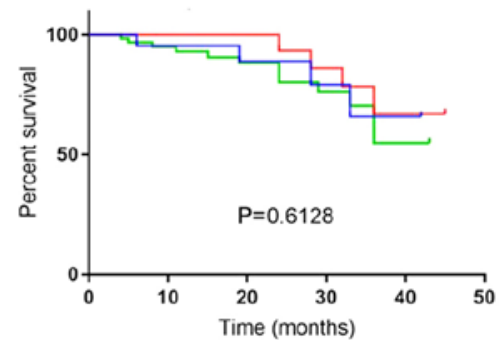

E

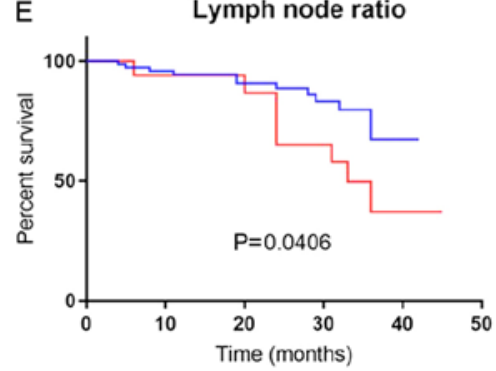

G

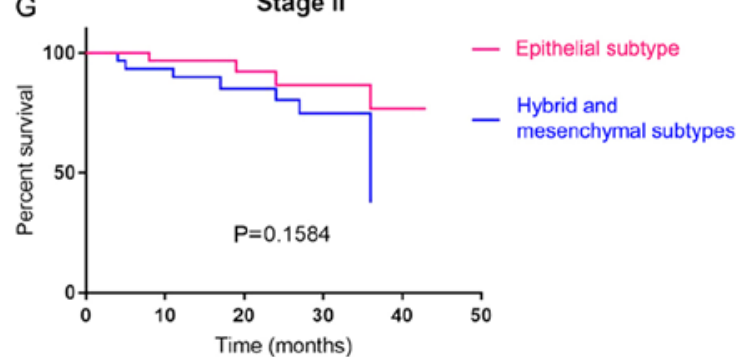

- Cytoplasm

- Nucleus \pm cytoplasm

- LNR $\leq 0.15$

- LNR $>0.15$
B

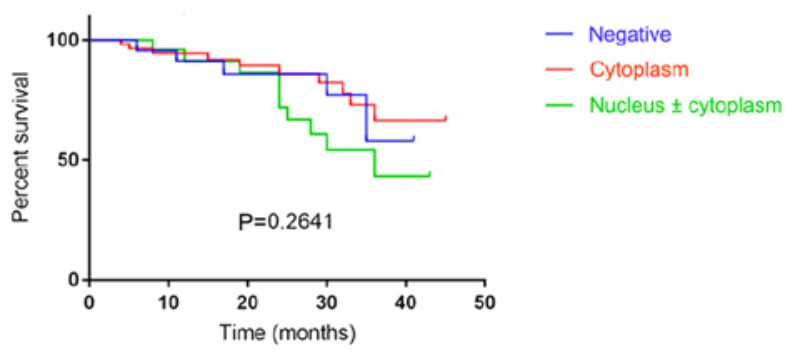

D Tumor budding grade

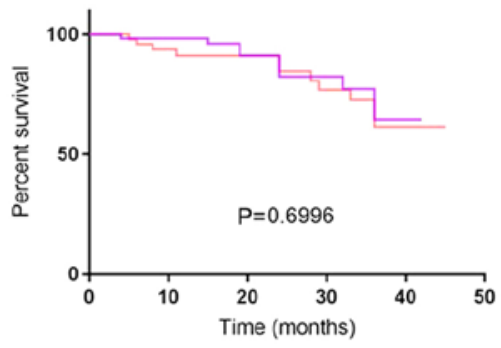

— Low-grade budding

— High-grade budding

F

Stage I
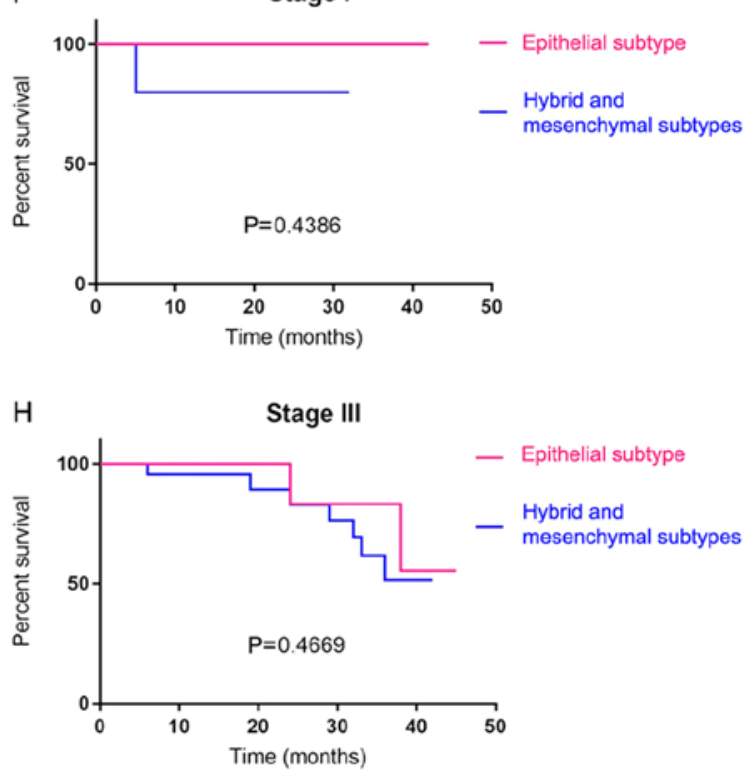

Figure 4. Examination of the independent prognostic value of clinicopathological factors by Kaplan-Meier curves. No independent prognostic value was observed for (A) molecular subtype, (B) subcellular localization of maspin in the tumor center, (C) subcellular localization of maspin in the invasion front or (D) the grade of tumor budding. (E) Lymph node ratio appeared to have a prognostic impact; however, no prognostic value was observed for the molecular classification, independent from the pathological stages: (F) stage I, (G) stage II or (H) stage III.

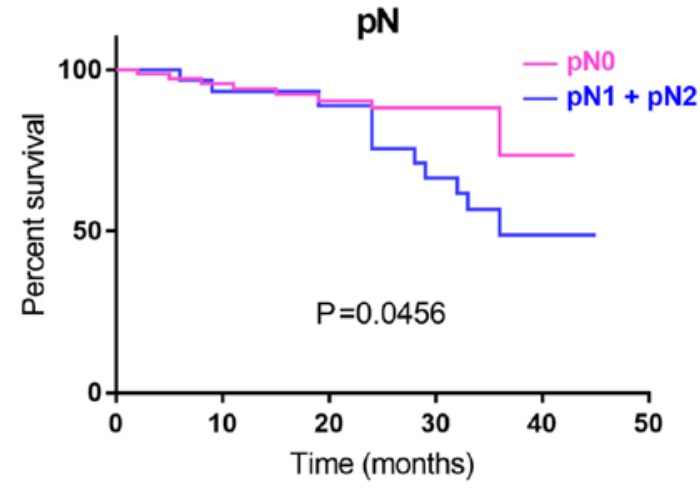

Figure 5. Kaplan-Meier curves indicate the independent prognostic value of the pN stage. pN, lymph node status.
In conclusion, the present study demonstrated that the proposed IHC panel represents a feasible option for the molecular classification of CRCs and a simpler way to guide case management. However, more complex studies in a larger cohort remain a necessity. The expression of maspin proves yet again to be a useful tool for identifying tumor buds and, through examining the subcellular localization of its staining, for evaluating EMT in CRCs, with nuclear positivity being an indicator of the mesenchymal subtype, lymph node metastasis, high-grade tumor budding and decreased OS. Epithelial-type CRCs with cytoplasmic expression of maspin are mostly non-metastatic, with a low-budding degree and longer OS. The most challenging cases remain the hybrid CRCs, whose particular behavior should be examined on a molecular level. 


\section{Acknowledgements}

The authors would like to thank Ms. Genoveva Rigmanyi (Research Center (CCAMF)-Microscopy Laboratory, University of Medicine, Pharmacy, Sciences and Technology, George Emil Palade) for her help in performing immunohistochemical and molecular stains.

\section{Funding}

The present study was supported by the University of Medicine, Pharmacy, Sciences and Technology of Tirgu Mures, Romania (grant no. 615/5/2019).

\section{Availability of data and materials}

The datasets used and/or analyzed during the present study are available from the corresponding author on reasonable request.

\section{Authors' contributions}

LB drafted the paper, designed the study and contributed to the immunohistochemical assessment. IJ contributed to the immunohistochemical assessment. TB performed the surgical interventions. ZF participated in the surgical interventions and patients' follow-up. PS participated in clinical and imaging investigation of patients, patient follow-up, database synthesis and literature review. IS participated in the clinical assessment of images, transdiciplinary management of patients and assessed data regarding lymph node ratio. CS participated in the histopathological and IHC assessment of the cases. SG undertook the study design, contributed in the histological and immunohistochemical assessment and conferred the final agreement for publication. All cases were managed by the same transdisciplinary team, which comprised radiologists (PS and IS), oncologic surgeons (TB and ZF) and pathologists (LB, IJ, CS and SG).

\section{Ethics approval and consent to participate}

Approval was obtained from the Ethical Committee of the University of Medicine and Pharmacy of Targu-Mures, Romania, for retrospective evaluation of the cases.

\section{Patient consent for publication}

Not applicable.

\section{Competing interests}

The authors declare that they have no competing interests.

\section{References}

1. Guinney J, Dienstmann R, Wang X, de Reyniès A, Schlicker A, Soneson C, Marisa L, Roepman P, Nyamundanda G, Angelino P, et al: The consensus molecular subtypes of colorectal cancer. Nat Med 21: 1350-1356, 2015.

2. Gurzu S, Silveanu C, Fetyko A, Butiurca V, Kovacs Z and Jung I: Systematic review of the old and new concepts in the epithelial-mesenchymal transition of colorectal cancer. World J Gastroenterol 22: 6764-6775, 2016.
3. Cao H, Xu E, Liu H, Wan L and Lai M: Epithelial-mesenchymal transition in colorectal cancer metastasis: A system review. Pathol Res Pract 211: 557-569, 2015.

4. Roseweir AK, Kong CY, Park JH, Bennett L, Powell AGMT, Quinn J, van Wyk HC, Horgan PG, McMillan DC, Edwards J and Roxburgh CS: A novel tumor-based epithelial-to-mesenchymal transition score that associates with prognosis and metastasis in patients with Stage II/III colorectal cancer. Int J Cancer 144: 150-159, 2019.

5. Gurzu S, Banias L, Kovacs Z and Jung I: Epithelial-mesenchymal transition of tumor budding in colorectal cancer: The mystery of CD44-positive stromal cells. Hum Pathol 71: 168-169, 2018.

6. Lugli A, Kirsch R, Ajioka Y, Bosman F, Cathomas G, Dawson H, El Zimaity H, Flejou JF, Hansen TP, Hartmann A, et al: Recommendations for reporting tumor budding in colorectal cancer based on the international tumor budding consensus conference (ITBCC) 2016. Mod Pathol 30: 1299-1311, 2017.

7. Ueno $H$, Ishiguro $M$, Nakatani $E$, Ishikawa $T$, Uetake $H$, Matsuda C, Nakamoto Y, Kotake M, Kurachi K, Egawa T, et al: Prospective multicenter study on the prognostic and predictive impact of tumor budding in stage II colon cancer: Results from the SACURA trial. J Clin Oncol 37: 1886-1894, 2019.

8. Banias L, Gurzu S, Kovacs Z, Bara T, Bara T Jr and Jung I: Nuclear maspin expression: A biomarker for budding assessment in colorectal cancer specimens. Pathol Res Pract 213: 1227-1230, 2017.

9. Banias L, Jung I and Gurzu S: Subcellular expression of maspin- from normal tissue to tumor cells. World J Meta-Anal 7: 142-155, 2019.

10. Gurzu S, Szentirmay Z, Popa D and Jung I: Practical value of the new system for Maspin assessment, in colorectal cancer. Neoplasma 60: 373-383, 2013.

11. Gurzu S, Szentirmay Z and Jung I: Molecular classification of colorectal cancer: A dream that can become a reality. Rom J Morphol Embryol 54: 241-245, 2013.

12. Gurzu S, Szentrimay Z, Toth E and Jung I: Possible predictive value of maspin expression in colorectal cancer. Recent Pat Anticancer Drug Discov 8: 183-190, 2013.

13. Amin MB, Edge S, Greene F, Byrd DR, Brookland RK, Washington MK, Gershenwald JE, Compton CC, Hess KR, Sullivan DC, et al: AJCC cancer staging manual. Springer 2017: 251-274, 2017.

14. Fulop ZZ, Gurzu S, Bara T, Dragus E, Bara T Jr, Voidazan S, Banias L and Jung I: Lymph node ratio, an independent prognostic factor for patients with stage II-III rectal carcinoma. Pathol Res Pract 215: 152384, 2019.

15. Luchini C, Bibeau F, Lightenberg MJL, Singh N, Nottegar A, Bosse T, Miller R, Riaz N, Douillard JY, Andre F and Scarpa A: ESMO recommendations on microsatellite instability testing for immunotherapy in cancer, and its relationship with PD-1/PD-L1 expression and tumour mutational burden; A systematic review-based approach. Ann Oncol 2019 (Epub ahead of print).

16. Kim WG, Kim JY and Park DY: Simple classifiers for molecular subtypes of colorectal cancer. Arab J Gastroenterol 18: 191-200, 2017.

17. Ten Hoorn S, Trinh A, de Jong J, Koens L and Vermeulen L: Classification of colorectal cancer in molecular subtypes by immunohistochemistry. Methods Mol Biol 1765: 179-191, 2018.

18. Lee CHA, Wilkins S, Oliva K, Staples MP and McMurrick PJ: Role of lymph node yield and lymph node ratio in predicting outcomes in non-metastatic colorectal cancer. BJS Open 3: 95-105, 2018.

19. Attaallah W, Gunal O, Manukyan M, Ozden G and Yegen C: Prognostic impact of the metastatic lymph node ratio on survival in rectal cancer. Ann Coloproctol 29: 100-105, 2013.

20. Kim JH, Cho NY, Bae JM, Kim KJ, Rhee YY, Lee HS and Kang GH: Nuclear maspin expression correlates with the $\mathrm{CpG}$ island methylator phenotype and tumor aggressiveness in colorectal cancer. Int J Clin Exp Pathol 8: 1920-1928, 2015.

21. Yamadera M, Shinto E, Kajiwara Y, Mochizuki S, Okamoto K, Shimazaki H, Hase K and Ueno H: Differential clinical impacts of tumour budding evaluated by the use of immunohistochemical and haematoxylin and eosin staining in stage II colorectal cancer. Histopathology 74: 1005-1013, 2019.

22. Hestetun KE, Brydøy M, Myklebust MP and Dahl O: Nuclear maspin expression as a predictive marker for fluorouracil treatment response in colon cancer. Acta Oncol 54: 470-479, 2015.

23. Santamaria PG, Moreno-Bueno G, Portillo F and Cano A: EMT: Present and future in clinical oncology. Mol Oncol 11: 718-738, 2017.

This work is licensed under a Creative Commons Attribution-NonCommercial-NoDerivatives 4.0 International (CC BY-NC-ND 4.0) License. 